\title{
ANALYSIS OF OPEN HEALTH DATA QUALITY USING DATA OBJECT-DRIVEN APPROACH TO DATA QUALITY EVALUATION: INSIGHTS FROM A LATVIAN CONTEXT
}

\author{
Anastasija Nikiforova \\ Faculty of Computing, University of Latvia \\ Rainis Boulevard 19. Riga, LV-1586, Latvia
}

\begin{abstract}
This research focuses on the analysis of the quality of open health data that are freely available and can be used by everyone for their own purposes. The quality of open data is crucial as it can lead to unreliable decision-making and financial losses, however, the quality of open health data has even more critical role. Despite its importance, this topic is rarely discussed. Therefore, the previously proposed data object-driven approach to data quality evaluation is applied to open health data in Latvia in order to (a) evaluate their quality, highlighting common quality issues that should be considered by both, users and data publishers, (b) demonstrate that the used approach is suitable for given purpose as it is simple enough, and ensures the involvement of users even without IT and data quality knowledge (domain experts) in the data quality analysis examining data for their own purposes. The proposed solution seems to be useful in establishing communication between data users and publishers, improving the overall quality of data.
\end{abstract}

\section{KEYWORDS}

Open Data, Health Data, Data Quality, Data Object-Driven Approach

\section{INTRODUCTION}

Data quality issue was the topic of interest for many years, and, nowadays, since data are everywhere, and their amount continuously increases, this issue is still popular and topical. One of the reasons of its topicality is the popularity of e-Government projects. However, despite the high number of open data sets all over the world, there is no confidence that published data are of high quality. More and more researches indicate the existence of data quality problems in open data. This issue is especially important in the case of health data, as it can lead to unreliable decisions, financial losses or even tragedies. As health and healthcare data are very broad concepts (Cabitza \& Batini, 2016), this research focuses on one subdomain - open health data. Despite open health data don't have sensitive data on people, i.e. patients, they are needed for health(care) planning and administrative purposes (Kerr et al., 2007). As the level of details of "open" data might be lower in comparison with "closed" data stored in databases, it is assumed quality checks could be simpler.

In addition, as open data are usually used by wide audience that may not have deep knowledge in IT or data quality areas, a solution that should be applied to open data sets should be simple enough ensuring particular users with possibility to take part in the analysis of open data. To achieve this aim previously proposed user-oriented data object-driven approach (Bicevskis, 2018a; Nikiforova, 2019) should be examined in order to ensure it is applicable on such data sets providing all necessary functionality making its quality analysis.

The paper is structured as follows: a short overview of the state of open [health(care)] data quality (Section 2), a description of data object-driven approach to data quality evaluation (Section 3), an analysis of open health(care) data (Section 4), conclusions (Section 5). 


\section{OPEN DATA QUALITY}

Nowadays, open data become more and more popular all around the world. As an example, European Data Portal https://www.europeandataportal.eu collects more than 800 thousand data sets. Moreover, open data significantly impact economy, as in accordance with (Tinholt, 2013), the aggregate economic impact from applications based on open data across the EU27 economy is estimated to be $€ 140$ billion annually. At the same time, the 2013 McKinsey Global Institute report estimated that open data could add over \$3 trillion annually in total value to the global economy (Castro et al., 2015). In accordance with G8 Open Data Charter, Open Government Data (OGD) impact economic growth, improving government services, reducing fraud and waste. It is obvious, that data quality has the crucial role in achieving these aims.

According to 8 principles of open data (SunlightFoundation, 2007), in order to admit data as open data they must be: complete; primary; timely; accessible; machine-processable; non-discriminatory; non-proprietary; licence-free. The main idea of every principle won't be discussed here, however, the main point is that these principles don't focus on data quality (Nikiforova, 2018a). The same tendency is observed in the case of evaluation of OGDs, as in accordance with (Klein et al., 2018), the quality aspect takes only the $4^{\text {th }}$ place by popularity after policy, benefit and risk in spite of the fact that quality can impact these aspects. At the same time, in accordance with European Data Portal, data quality appears as one of most problematical dimensions for open data portals.

Importance of data quality was discussed for multiple times and, unfortunately, the results are disappointing. The list of researches indicating the existence of data quality problems in open data is quite impressive (Ferney et al., 2017; Kerr et al., 2007; Kuk and Davies, 2011; Martin, 2014; Nikiforova, 2018a, 2018b; Nikiforova and Bicevskis, 2019; Vetrò et al., 2016 etc.).

\subsection{The State of Open Data in Latvia}

In 2017 the Latvian Ministry of Environmental Protection and Regional Development has launched the new Latvian Open Data Portal (European Data Portal, 2018) - data.gov.lv. At the moment of its launch it had 33 data sets from 13 data publishers, in July of 2018 it had 139 data sets from 41 publishers (Nikiforova, 2018b), but in June of 2019 - 217 data sets from 62 publishers. It demonstrates how quickly the number of data sets increases. Moreover, it isn't the only one prove of Latvian OGD development and progress.

Latvia is one of 70 countries participating in the Open Government Partnership (2017) - an international platform for domestic reformers that committed to making their governments more open, accountable, and responsive to citizens. At the same time, in accordance with Open Data Maturity report (European Data Portal, 2018), that measures open data maturity across Europe, Latvia belongs to the fast-trackers (among beginners, followers, fast-trackers, trend-setters). Moreover, Latvia has the highest rate of open data maturity in comparison with neighbourhoods from Baltic States and Scandinavian countries. Furthermore, in recent years open data maturity of Latvian open data portal continuously improves, as in 2016 Latvia was ranked $31^{\text {st }}$, in $2017-20^{\text {th }}$, however in $2018-$ the $12^{\text {th }}$. As for the quality aspect, that was added to the series of indicators only in 2018 data, Latvia takes the $11^{\text {th }}$ place with 370 of 520 points. An important point is that in comparison with average rate for all analysed countries, the state of the quality of open data maturity for Latvia is the worst aspect among impact, policy, portal, and quality (only 62\% while the average is $71 \%$ ).

\subsection{Open Health(care) Data}

In last decades, the volume of health(care) data continuously increasing, and it is expected to grow dramatically in the years ahead (Raghupathi, 2014). Nowadays, open health data is one of the most popular categories of open data, and, according to European Open Data portal, in Europe it takes the $6^{\text {th }}$ place among 13 categories. As for Latvia, health data take the $6^{\text {th }}$ place among 14 categories, however, in 2014 they took the $7^{\text {th }}$ place among 9 categories (Bojārs and Liepinšs, 2014). It means that their popularity in Latvia increases.

Aims and possible uses of open health(care) data can be very different, as health data and information are characterized by multiple number of possible applications, uses and users (Cabitza and Batini, 2016). Some of them can be compared with (Schmidt et al., 2015) as open health data can (1) form the basis for health and medicines authority's hospital statistics, or health economic calculations, (2) provide authorities with data to support hospital planning, (3) provide data to support the authorities responsible for hospital inspection, 
(4) monitor the frequency of various diseases and treatments, (5) provide a sampling frame for medical research, (6) facilitate quality assurance of the health(care) services etc.. Moreover, according to Andreassen et al., (2007), the US studies have found that between 56\% and 79\% of Internet users seek health information online. For Latvia this proportion is $35 \%$, for Poland - $42 \%$ with the lowest proportion of Internet health users in the Southern countries - 30\% in Portugal and 23\% in Greece. It means that health(care) data must be of high quality, as in spite of the fact that some open health(care) data sets contain mainly numerical data that, possibly, doesn't make sense to common user, there are such data sets that can be very useful searching data on medications, their dose, contraindications and other information available for the wide audience.

Despite data quality has crucial role, the current state of the [open health(care)] data quality is not reassuring. As an example, the existence of data quality issues was detected in the Danish National Patient Registry (Schmidt et al., 2015), even in spite the authors analysed data set using only two measures (dimensions), namely validity and completeness. Moreover, despite analysed data were "closed", identified data quality problems are common for "open" data, too. The list of researches discussing quality of health(care) data in many countries such as New Zealand - (Kerr et al., 2007), (Raghupathi, 2014), Denmark - (Schmidt et al., 2015), Brazil - (Oliveira, 2016), USA, UK and Japan - (Yi, 2019), Switzerland - (Wanner et al., 2018), Sweden - (Tomic, 2015), Colombia - (Prieto Rodríguez, 2018), comes to the one conclusion - health(care) data have data quality problems (with the sole exception of Norway - (Larsen, 2009)).

\section{DATA OBJECT-DRIVEN APPROACH TO DATA QUALITY EVALUATION}

The use of open data brings significant benefits data providers as because of multiple number of possible use-cases, data users address various challenges that can rarely be solved by data providers alone. As a result, a simple and constructive approach to data quality evaluation is needed in order to provide users with possibility to take part in open data analysis even if they don't have deep knowledge in IT and data quality areas. The data object-driven approach to data quality evaluation previously proposed in (Bicevskis, 2018a; Nikiforova, 2019) is considered as highly appropriate for given purpose.

It doesn't use "quality dimension" concept, that became traditional for data quality solutions, as there is no universal classification that should be followed by data quality solutions' developers and users, despite the high number of attempts. In accordance with Scannapieco et al. (2005), even if there is agreement on dimensions that are universally important, there is no agreement on their meanings. In different proposals, the same name is often used to indicate semantically different things and vice versa. In addition, each dimension can be supplied with one or more metrics that varies from one solution to another. As a result, the number of different data quality dimensions, their definitions and grouping are often useful for only particular solution. As an example, Kerr et al. (2007) analyses the quality of New Zealand's healthcare data, and despite they admit the problem of different meanings assignation to the same dimensions, they choose 6 data quality dimensions, supplying them with 24 characteristics and 69 data quality criteria. Such high number of criteria defined by authors minimise a possibility that this solution will be used, especially by non-IT and non-DQ experts. Moreover, while some researches propose too high number of dimensions, certain researches limit them to accuracy and completeness ignoring other dimensions (Dahbi et al., 2018; Weiskopf and Weng 2013). The proposed approach doesn't respect such limitations and provides possibility to define data quality requirements that depends on the use-case. Taking into account the common data quality division in syntactic and semantic accuracy (Scannapieco, et al., 2005) as well as multiple issues appearing from "quality dimension" concept, the proposed approach avoids it, respecting wider concept "data quality requirement" where "data quality dimension" is its subset. Syntactic accuracy of the proposed solution can be found in (Bicevskis et al., 2018a; Nikiforova, 2018b), however the semantic accuracy was presented in (Nikiforova and Bicevskis, 2019). As the comprehensive discussion on the existing solutions, rationale and detail of the given approach were discussed previously, they won't be discussed in detail here, explaining only the basics of the solution that should be understood by the users.

The data object-driven approach consists of 3 main components: (1) data object, (2) quality requirements and (3) quality measuring process. All components are defined using graphical flowchart-based diagrams, that are based on the developed Domain Specific Languages (DSL) that are easy to read, interpret and modify 
even for non-IT experts. In scope of particular research, these components are demonstrated by the example of one of analysed data sets - Medicinal Product Register of Latvia.

Data object defines data which quality should be analysed. Two types of data objects are possible: (a) primary - data object which quality is analysed, (b) secondary - data object against which primary data object can be analysed ensuring contextual/ semantic analysis of primary data object (Nikiforova and Bicevskis, 2019). Data object "Medicinal_Product" is depicted with 9 of 41 parameters (Figure 1). It is primary data object which quality is analysed against three secondary data objects, namely, "Country", "Country_LV" and "ATC", that are provided by other open data publishers.

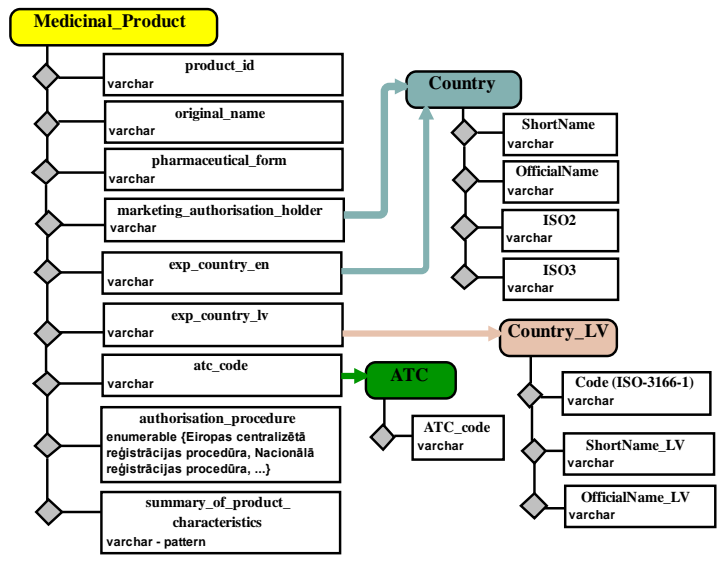

Figure 1. Data object (DO) "Medicinal Product"

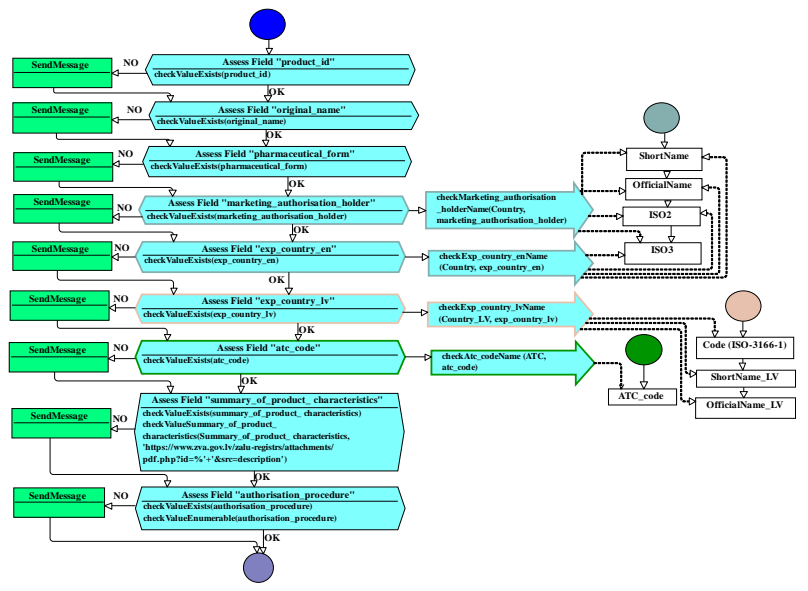

Figure 2. Quality specification for DO "Medicinal Product"

As for data quality specification, conditions that must be met in order to admit data as qualitative, are formulated using logical expressions where operand of every condition is a name of the data object's attribute (Figure 2). Data quality specification as well as data object can significantly vary depending on the use-case. Data quality conditions are formulated for both, attributes in context of one data object, and attributes in context of multiple data objects, depicting relations of appropriate attributes by arrow with logical condition on how these attributes are interconnected. Every parameter of the primary data object can be related to an arbitrary number of parameters of the secondary data object.
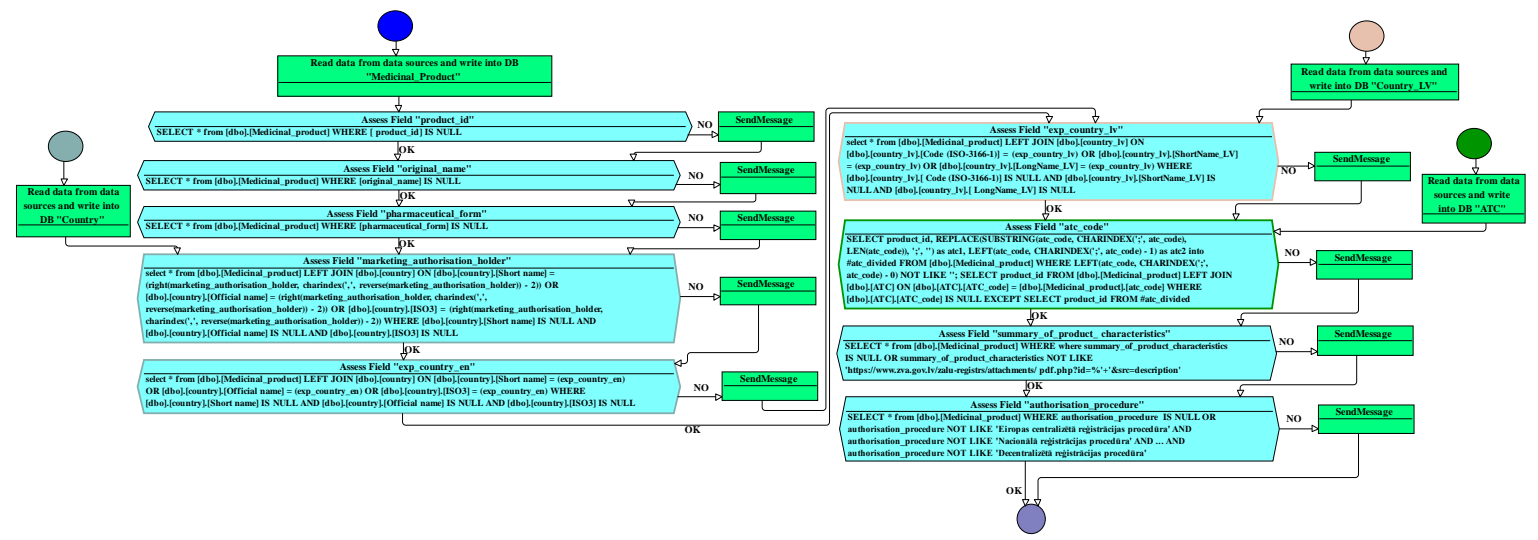

Figure 3. Data quality evaluation process of data object "Medicinal Product"

The last step is description of quality measuring process (Figure 3). It defines a procedure that must be performed to evaluate data quality, from data reading from a data source and their loading to database for further processing to replacing of quality conditions defined at the previous step by executable SQL queries (or another executable artefact, as its nature depends on the skills, experience and preferences of a user involved at this stage). This step is supposed to be done by an IT-expert. The result of the execution of this diagram is a protocol containing all records that don't conform defined quality requirements. 


\section{DATA QUALITY ANALYSIS OF OPEN HEALTH(CARE) DATA}

All open health(care) data sets in Latvia were firstly published in 2018 and now they are represented by 15 data sets from 7 different data publishers (the $1^{\text {st }}$ and $2^{\text {nd }}$ columns in Table 1).

Despite the part of data sets is supplied with metadata, specified by CKAN, the majority of data publishers do not fill all metadata or do not use standard values for them as only 8 out of 15 data sets are supplied with explanation of parameters. This issue is typical not only for Latvia but also for other countries, for instance, Brazil (Oliveira, 2016). Moreover, only 6 out of 15 data sets are updated as frequently as it is promised. Possibly, identified problem occurs because by the term "frequency of updates" data publishers understand the frequency of data updates in their systems instead of data publishing as open data sets. This issue is also widely spread among other countries, as, according to Tinholt (2013), 22 out of 23 analysed countries and even the UK (Kuk and Davies, 2011) publish data that are not regularly updated.The most popular open data format in Latvia is .xlsx as $53.3 \%$ of data sets are provided in this format, while $26.7 \%$ in .zip archive including data sets in . $x l s x$ and .csv format, and 1 data set in .html that cannot be considered open data in the traditional meaning as it is link on the statistics ("Database of official statistics"). The positive point is that almost all available data sets are provided in machine-readable format.

As for data quality, that is the focus of the current research, every parameter of every data set was analysed in order to make comprehensive data quality analysis. Apart 15 primary data objects, 11 secondary data objects were involved in data quality analysis and applied on 35 parameters of primary data objects.

The most popular and frequently occurred data quality issues in analysed data sets are: (a) contextual data quality issues, (b) empty values (completeness); (c) multiple notation for the same object in scope of one data object and even parameter; (d) issues in interrelated parameters. Table 1 provides an overview of these data quality issues by number of parameters in which they occur for every data set.

Table 1 . The most common data quality problems by data set

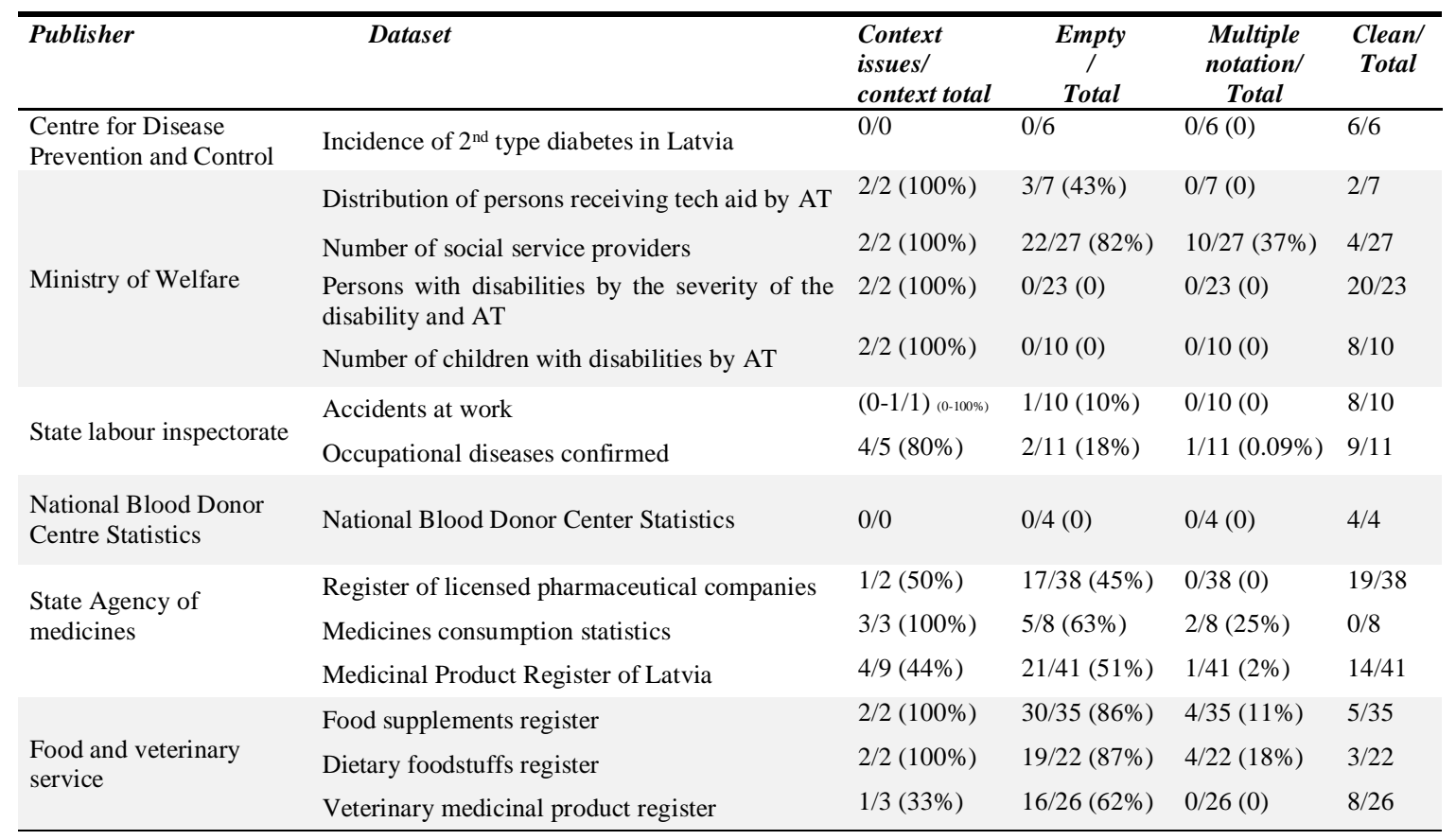

One of the most commonly appeared data quality issue was empty values (the $4^{\text {th }}$ column of Table 1 ). More precisely, 136 out of $167(81.4 \%)$ analysed parameters at least one value was empty. The number of empty values per data set and parameter varies from 1 to all values of a certain parameter. However, the total number of empty values in analysed data sets is $15 \%$. In some cases, problem of empty values appears for the primary data of the data sets, for instance, in the case of "Dietary foodstuffs register", 4 records don't have [Name] and [ProducerName] despite these parameters are primary for a particular data object. However, sometimes, an empty value may be allowed, for instance, in the case of the "Food supplements register", the 
[Reason for cancellation] parameter must have value only if the value of parameter [Status] is "cancelled". In other words, if a parameter should have value only when some condition is true, or it is not mandatory, it may not be considered a data quality problem (depending on the use case). 28 of 136 detected empty values may not be considered as data quality issues, however, while there are no any notes from the data publisher regarding the nullability of these parameters, there is no certainty, that there are no any problems there, as empty values may have different interpretations. The issue of completeness is almost "traditional" and it occurs not only in the case of open data (Oliveira, 2016; Yi, 2019) but also in "closed" data (Schmidt et al., 2015; Tomic, 2015) in many sectors and countries (Wanner et al., 2018). However, some researches demonstrate a high level of data completeness can be achieved - Cancer Registry of Iceland (Sigurdardottir, 2012) and Norway (Larsen, 2009) (however, both are examples of "closed" data).

One more widely occurred data quality problem is multiple notations for a single object within a single data set and even a parameter (the $5^{\text {th }}$ column of Table 1). This problem occurs in 6 out of 15 data sets $(40 \%)$ - in 22 out of 167 parameters (13.2\%). It may appear in different ways such as a different name: (a) for one country, (b) for the type of preparation, ingredient or unit size, (c) for different patterns for one value, for instance, phone or registration number (with or without (1) code or (2) delimiter; type of delimiter etc.). For 2 data sets it is represented by two different notations indicating the absence of a value: $N U L L$ and ' $O$ ' (often called "heterogeneity"). On the one hand, this data quality problem could be considered as insignificant as the value stored in the appropriate field may point on an absence of value. However, there is no confidence that both values $N U L L$ and ' $O$ ' have the same meaning, as ' $O$ ' can point out to the value that is equal to zero, while NULL can mean that the value isn't known. It means, that even if the user has noticed the presence of both of these values, it is difficult to make a decision on how to process these data. As for "type of preparation, ingredient, or unit size", one of the most expressive examples are values of [Component] and [Measure] parameters, where the same value can appear in multiple ways: (a) singular, (b) plural, (c) shortened form, (d) some of the previous forms but with a spelling mistake. Moreover, while this problem occurs 8 times ( 2 of them relates to multiple values denoting value doesn't exist), in 5 cases it could be solved, involving the mechanisms, controlling the list of permissible values. At this point, users, inserting data, choose value according to their viewpoint. This problem is also widely spread for many sectors and even countries, and one of the most expressive examples is OGD of the UK (Kuk and Davies, 2011).

As for the contextual data quality analysis within multiple data objects ( $3^{\text {rd }}$ column in Table 1 ), only 1 data set among 12 analysed (8.3\%) didn't had any data quality issues ("Accidents at work"), however, some manipulations were needed in order to achieve this result. The data set "Accidents at work" was analysed against the secondary data object ("Work codes") in order to check the correctness of provided codes by comparing them with the codes conforming the standard. When this check was made for the first time, comparing the codes in both data sets, it appeared, that the values of the [Occupation of the injured person (code)] parameter don't correspond to the work codes, until the moment when the patterns of both values from both data sets were analysed and compared. The pattern of the values of the analysed data set was modified: the code value "88.3332-03" corresponds to the values "8332" - "03" (two parameters) in the secondary data object. In result of this comparison, any quality problems weren't detected, however, it was a relatively complex check as the comparison of these data sets can be performed only after multiple modifications of the parameters' values. As for data quality, such manipulations should be avoided, ensuring homogeneity between data sets. Another example relating to this category, is the case of 4 data sets published by the Ministry of Welfare, where 3 records have data quality issues in the [ATTU code] and [City, county] parameters. These parameters are supposed to store the code of the administrative territory and city that must correspond to the secondary data object "Classification of Administrative Territories and Territorial Units". However, 3 values of the primary data objects are invalid - aren't available in the secondary data set. However, possibly, the data publisher is aware of this data quality problem, as the appropriate values of the [City, county] parameter - "Total", "Abroad" and "Address isn't specified" make sense. Unfortunately, the fact that data publisher is aware of this issue doesn't appear, as, even if he knows about it, he should inform the data consumers about it, at least by making notes about it together with a description of the data sets' parameters. In other cases, this data quality problem can be easily unnoticed and can lead to inaccurate data analysis results. One more observation is that in spite that the codes in the primary and secondary data objects can be the same, the name corresponding to these codes can vary depending on the data set. However, the difference in names may occur just in notation rather than in the meaning. As an example, "Occupational diseases confirmed" data set was analysed against three secondary data objects. The number of quality defects in [Classification of Occupational Disease (code)] parameter is 5, however, in [Classification of 
Occupational Disease - 871 . While 5 of them are invalid as the code is invalid, 866 out of 871 values can be admitted as of low quality only in the cases, when values should be equal one to one. However, the other 10 out of 15 data sets have more significant data quality problems, storing values that don't correspond to secondary data objects. In total 25 out of 35 parameters $(71.4 \%)$ had at least few data quality issues.

Another problem (or possibly just anomaly) was detected in the "Number of social service providers" data set in the case of three parameters - [Service with accommodation] and [Service without accommodation] and [Service with and without accommodation]. From the users' viewpoint, it is almost obvious, that the total number of [Service with accommodation] and [Service without accommodation] for every particular record must be equal with [Service with and without accommodation] value, however, for 95 records this assumption is not in force. There are at least two possible explanations of such result: (1) there are 95 data quality problems; (2) these field aren't interconnected, and the sum of values of the first two parameters not necessarily should be equal with the value of the third parameter. However, as information on how these values were calculated isn't provided, it is not possible to understand, which of these options is correct. A similar anomaly was observed in the "Number of children with disabilities by administrative territory" data set. In accordance with the principles of open data, data publishers must provide a brief explanation of the parameters and how numerical data was gotten, in order to avoid such misunderstandings. Another problem appearing for 4 out of 15 data sets $(26.7 \%)$ is the different number of interrelated values. It may appear in different ways: (a) values in different languages, (b) ID number and name, (c) name and supplementary data such as type, country, phone number of representatives.

Few inaccuracies appeared in the result of analysis of the 3 data objects relate to an inaccurate choice of the values delimiter in the .csv files listing more than one value per parameter. The delimiter that separates multiple values of one parameter is the same with delimiter of parameters. As a result, the processing of these data sets became more complicated as it was necessary to understand in which cases the delimiter separates multiple values of one parameter, and in which - multiple parameters, since both types of delimiters are the same. It is highly recommended to use different delimiters for parameters and multiple values of one parameter. This issue is also common for open data sets of many countries (Kuk et al., 2011).

\section{CONCLUSION}

Despite the importance of data quality, the quality of open data is not always one of the main areas of analysis and evaluation of open data. The paper demonstrates and summarizes a quality analysis of Latvian open health(care) data, highlighting commonly appeared data quality problems and some specific problems that should be avoided by data publishers and taken into account by data users. Unfortunately, results of the research demonstrate open health(care) data have a high number of different data quality problem, however, data publishers (who provides data used in their IS), probably, don't even aware of them. The most frequently occurred of them are: (a) contextual data quality issues, (b) empty values even for primary data; (c) multiple denotation for the same object within one data object and even a parameter; (d) issues on interrelated parameters. These quality issues become a tendency for the most part of the analysed items.

Obviously, such an analysis and use of a data object-driven approach to data quality evaluation can be applied not only to open health(care) data but also to other structured and semi-structured data - this solution is effective in many domains. The advantages of the used approach are that (a) it can be applied to "foreign" data sets without any information on how data were accrued and processed - it is an external mechanism with a higher level of abstraction, (b) it can be used even by users without IT and DQ knowledge that is highly important in order to improve data quality by increasing participation and inherent feedback on the quality of data sets (corresponds with (Attard et al., 2015; Ruijer et al., 2019)). Moreover, the quality of data in this way can be improved not only at the national level, but also at the international level as it was already demonstrated in (Bicevskis et al., 2018b, Nikiforova and Bicevskis, 2019).

\section{ACKNOWLEDGMENT}

This work has been supported by University of Latvia Faculty of Computing project AAP2016/B032 "Innovative information technologies". 


\section{REFERENCES}

Agrawal, S. et al., 2013. A survey of Indian open data. In Proceedings of the 5th IBM Collaborative Academia Research Exchange Workshop (p. 2). ACM.

Andreassen, H. et al., 2007. European citizens' use of E-health services: a study of seven countries. BMC public health.

Attard, J. et al., 2015. A systematic review of open government data initiatives. Government Information Quarterly.

Bicevskis, J. et al., 2018a. An Approach to Data Quality Evaluation. In 2018 Fifth International Conference on Social Networks Analysis, Management and Security (SNAMS) (pp. 196-201). IEEE.

Bicevskis, J. et al., 2018b. Data quality evaluation: a comparative analysis of company registers' open data in four European countries. In Communication Papers of the FedCSIS (pp. 197-204).

Bojārs, U., Liepiňš, R., 2014. The State of Open Data in Latvia: 2014. arXiv preprint arXiv: 1406.5052.

Cabitza, F., Batini, C., 2016. Information quality in health(care). In Data and Information Quality, Springer, Cham.

Castro, D. Korte, T., 2015. Open Data in the G8: A Review of Progress on the Open Data Charter.

Dahbi, K. Y. et al., 2018. Toward an Evaluation Model for Open Government Data Portals. In International Conference Europe Middle East \& North Africa Information Systems and Technologies to Support Learning. Springer, Cham.

Ferney, M. M. J. et al., 2017. Assessing data quality in open data: A case study. In 2017 Congreso Internacional de Innovacion y Tendencias en Ingenieria (CONIITI) (pp. 1-5). IEEE.

Kerr, K. et al., 2007. Data quality information and decision making: a health(care) case study. ACIS 2007 Proceedings.

Klein, R. H., et al., 2018. Open government data: concepts, approaches and dimensions over time. Revista Economia \& Gestão, 18(49), 4-24.

Kuk, G., Davies, T., 2011. The roles of agency and artifacts in assembling open data complementarities.

Larsen, I. K. et al., 2009. Data quality at the Cancer Registry of Norway: an overview of comparability, completeness, validity and timeliness. European journal of cancer, 45(7), 1218-1231.

Martin, C., 2014. Barriers to the open government data agenda: Taking a multi-level perspective. Policy \& Internet, 6(3).

Nikiforova, A., Bicevskis, J., 2019. An Extended Data Object-driven Approach to Data Quality Evaluation: Contextual Data Quality Analysis. In 21th International Conference on Enterprise Information Systems, ICEIS 2019.

Nikiforova, A., 2018a. Open Data Quality. In Baltic DB\&IS 2018 Joint Proceedings of the Conference Forum and Doctoral Consortium, Trakai, Lithuania (Vol. 2158, pp. 00742158-1).

Nikiforova, A., 2018b. Open Data Quality Evaluation: A Comparative Analysis of Open Data in Latvia. BJMC, 6(4).

Oliveira, M. I. S. et al., 2016. Open government data portals analysis: The brazilian case. In Proceedings of the 17th International Digital Government Research Conference on Digital Government Research (pp. 415-424). ACM.

Open Government Partnership, 2017. Latvia National Action Plan 2015-2017. https://www.opengovpartnership.org/documents/latvia-national-action-plan-2015-2017-updated-changes

Prieto Rodríguez, J. D., Suárez Hurtado, V., 2018. Medical Records Digital Perspective Colombian: Safety, Quality, and Management of Data. Journal of Alternative Perspectives in the Social Sciences, 9(3).

Raghupathi, W., Raghupathi, V., 2014. Big data analytics in health(care): promise and potential. Health information science and systems, 2(1), 3.

Ruijer, E., Meijer, A., 2019. Open Government Data as an Innovation Process: Lessons from a Living Lab Experiment. Public Performance \& Management Review, 1-23.

Scannapieco, M. et al., 2005. Data quality at a glance. Datenbank-Spektrum, 14(January), 6-14.

Schmidt, M. et al., 2015. The Danish National Patient Registry: a review of content, data quality, and research potential. Clinical epidemiology, 7, 449.

Sigurdardottir, L. G. et al., 2012. Data quality at the Icelandic Cancer Registry: comparability, validity, timeliness and completeness. Acta oncologica, 51(7), 880-889.

SunlightFoundation, 2007. Ten Principles For Opening Up Government Information.

Tinholt, D., 2013. The Open Data Economy: Unlocking Economic Value by Opening Government and Public Data. Capgemini Consulting.

Tomic, K. et al., 2015. Evaluation of data quality in the National Prostate Cancer Register of Sweden. European journal of cancer, 51(1), 101-111.

Wanner, M. et al., 2018. Indicators of data quality at the cancer registry Zurich and Zug in Switzerland. BioMed research international.

Weiskopf, N. G., Weng, C., 2013. Methods and dimensions of electronic health record data quality assessment: enabling reuse for clinical research. Journal of the American Medical Informatics Association, 20(1), 144-151.

Yi, M., 2019. Exploring the quality of government open data: Comparison study of the UK, the USA and Korea. 